\title{
Tubeless versus common minimally invasive percutaneous nephrolithotomy for renal stones under local anesthesia: analysis of clinical outcomes
}

\section{GenYi Qu}

The Affiliated ZhuZhou Hospital XiangYa Medical College of Central South University

Yong Xu ( $\nabla$ tigerhnllxu@126.com )

The Affiliated ZhuZhou Hospital XiangYa Medical College of Central South University

Cheng Tang

The Affiliated ZhuZhou Hospital XiangYa Medical College of Central South University JinGe Liu

The Affiliated ZhuZhou Hospital XiangYa Medical College of Central South University HaiBo Nie

The Affiliated ZhuZhou Hospital XiangYa Medical College of Central South University

\section{XiuFang Jiang}

The Affiliated ZhuZhou Hospital XiangYa Medical College of Central South University

\section{WenLin Huang}

The Affiliated ZhuZhou Hospital XiangYa Medical College of Central South University

\section{Guang Yang}

The Affiliated ZhuZhou Hospital XiangYa Mediacal College of Central South University

\section{Research article}

Keywords: renal stones, local anesthesia, tubeless, minimally invasive percutaneous nephrolithotomy

Posted Date: April 30th, 2020

DOI: https://doi.org/10.21203/rs.3.rs-22569/v1

License: (9) This work is licensed under a Creative Commons Attribution 4.0 International License. Read Full License 


\section{Abstract}

Background: To compare the clinical outcomes of tubeless minimally invasive percutaneous nephrolithotomy $₫ \mathrm{mPCNL}$ \and common minimally invasive percutaneous nephrolithotomy under local anesthesia, and to explore the feasibility, safety and clinical efficacy of tubeless MPCNL under local anesthesia.

Methods: Patients with PCNL who underwent local anesthesia from January 1, 2018 to November 30, 2018. The patients were divided into Group 1 (tubeless mPCNL: indwelling double $\mathrm{J}$ tube, no indwelling nephrostomy tube) and group 2 (common $\mathrm{MPCNL}$ : indwelling double $\mathrm{J}$ tube and nephrostomy tube). The intraoperative and postoperative parameters of the two groups were compared, including operation time, average postoperative hospital stay, puncture position, postoperative hemoglobin decline, postoperative visual analogue scale (VAS), analgesic use, postoperative fever and stone removal rate.

Results: In group 1,the VAS scores of 6 hours after surgery was $4.07 \pm 1.79$ significantly lower than that for group $2(4.07 \pm 1.79$ vs $6.24 \pm 1.33, P<0.05)$. and There was a statistically significant difference in the use of analgesics between the two groups $\otimes P<0.05 \rrbracket$. The VAS scores of day 1 after surgery $(2.50 \pm 1.76 \mathrm{vs}$ $3.83 \pm 2.22$ in group 1 and group2) was significantly lower in the tubeless mPCNL $(P<0.05)$. The hospital stay for group 1 was significantly shorter than group $2(3.15 \pm 1.42$ vs $6.48 \pm 1.88$ days, $P<0.01)$ ). There were no significant differences in the operation time, puncture position, postoperative hemoglobin decline rate, VAS score on the day of discharge, postoperative fever, and stone removal rate between the two groups $(P>0.05)$. Conclusion: Tubeless $\mathrm{mPCNL}$ is a simple, safe and effective technique under local anesthesia. It is worthy of clinical application. Tubeless $\mathrm{MPCNL}$ treatment for renal stones can significantly reduce postoperative pain and shorten hospital stays compared with common m PCNL.

\section{Background}

PCNL has now become one of the preferred treatments for renal stones and some of the upper ureteral calculi. The EAU Guidelines recommend PCNL as a first-line treatment for renal stones larger than $20 \mathrm{~mm}^{[1]}$. However, the drainage method after PCNL is still being improved and improved continuously ${ }^{[2]}$. The traditional drainage method after PCNL is indwelling renal fistula and ureteral stent. In recent years, tubeless PCNL (that is, only the ureteral stent tube is not placed after the operation) has attracted more and more people's attention. It has been published that tubeless PCNL can significantly reduce patient's postoperative pain and discomfort ${ }^{[3,4]}$. Besides, tubeless PCNL has the potential advantage of shorter hospital stay ${ }^{[3,4]}$. However, the surgical inclusion criteria are so strict that this method has not been widely accepted and popularized. for PCNL can be performed under both general anesthesia and local anesthesia. Recently, studies have reported that PCNL is superior in local anesthesia than in general anesthesia including lower dose requirements for analgesics ${ }^{[5,6]}$. In this study, the feasibility, efficacy and safety of tubeless PCNL under local anesthesia were studied by retrospective trials, providing further evidence-based medical evidence for the development of tubeless PCNL. 


\section{Methods}

\subsection{Patients selection}

Between January 1, 2018 and November 30, 2018, 135 patients undergoing PCNL were incorporated into this retrospective study at our center, including 68 patients underwent tubeless PCNL (group 1, including 46 males and 22 females) and 67 patients common mPCNL (group 2, including 41 males and 26 females). The inclusion criteria were intravenous urography (IVU) or CT diagnosis of non-complex kidney stones. The stones diameter was $20 \sim 40 \mathrm{~mm}$. The exclusion criteria were multi-channel PCNL, intraoperative aspiration system perforation, severe bleeding, urinary tract infection, renal empyema, renal stones with renal cortical thickness $<5 \mathrm{~mm}$, residual stones requiring secondary PCNL, ureteral stricture or renal pelvis, stenosis of the ureteral junction, previous history of renal surgery, cardiopulmonary and pulmonary infections, mental disorders, coagulopathy, anesthesia allergy, spinal deformity. This study has been approved by the Affiliated ZhuZhou Hospital XiangYa Medical College CSU, a ethics number (NO:2017-08116). All participating patients have signed surgical consent, anesthesia informed consent and clinical research consent. This study was conducted in accordance with the Declaration of Helsinki and good clinical practice according to the International Conference on Harmonization guidelines.

\subsection{Surgery methods}

Intramuscular injection of meperidine hydrochloride $75 \mathrm{mg}$ and promethazine hydrochloride $25 \mathrm{mg}$ was given 30 min before surgery. $10 \mathrm{ml}$ of $1 \%$ lidocaine hydrochloride was pushed through the urethra with a syringe at lithotomy position. After 5 minutes, the $5 \mathrm{~F}$ ureteral catheter was retrogradely inserted from the ureter to the renal pelvis under the ureteroscope. The ureteral catheter was connected with saline to establish artificial hydronephrosis, and the catheter was indwelled and fixed. With the patient in a prone position, the point and direction of puncture under the 11th intercostal space or the 12th rib were determined under ultrasonic positioning. Local anesthesia was performed with $10 \sim 40 \mathrm{ml}$ of $1 \%$ lidocaine hydrochloride. For the estimated longer operation time, $0.1 \mathrm{ml}$ of epinephrine was added per $10 \mathrm{ml}$ of $1 \%$ lidocaine hydrochloride, and the amount of an aesthetic was not more than $40 \mathrm{ml}$. After successful anesthesia, under the guidance of B-ultrasound, the correct position of $18 \mathrm{G}$ puncture needle was confirmed, Ultrasonography-guided percutaneous punctures with an $18 \mathrm{G}$ puncture needle were made into the designed calix. After the urine efflux was seen, a guidewire (PTFE-coated guidewire; Coloplast, Humlebæk, Denmark) was inserted into the collecting system. A 1-cm skin incision was made, use dilation stepwise system to expand to $18 \mathrm{~F}$ in turn, and a $18 \mathrm{~F}$ working sheath was placed directly as the percutaneous tract. Under direct nephroscope (8.5/11.5F) (Karl Storz GmbH $\square$ Co.KG,Tuttlingen $\square$ Germany)vision, the stone was fragmented by holmium laser. The stone fragments were pushed out by an endoscopic pulsed perfusion pump or taken out by lithotomy forceps. A kidney ultrasound and X-ray was performed a second time to confirm the clearance of the stone before the end of the operation. Finally, a 5F double-J stent was inserted in the ureter, and a nephrostomy tube (18F) was 


\subsection{Patients records}

The patient's age, gender, height, weight, stone size, operation time, the average postoperative hospital stay, puncture location, postoperative hemoglobin decline, postoperative visual analogue scale (VAS), postoperative fever, and stone removal rate were observed in this study. A comparative study was performed on the parameters of the two groups of patients.

\subsection{Statistical analysis methods}

Statistical analysis was performed using the SPSS 22.0 statistical software package. The continuous variables were expressed as mean \pm standard deviation $(x \pm s)$. The measurement data were analyzed by $t$ test. The qualitative data were analyzed by chi-square test. $P<0.05$ was considered statistically significant.

\section{Results}

\subsection{Comparison of demographic data and stone characteristics of the two groups}

The general conditions and stone characteristics of the two groups were shown in Table 1. There were no significant differences in age, gender, BMl, stone size and stone position between the two groups $(P>$ 0.05).

Table 1

Comparison of general clinical data between the two groups

\begin{tabular}{|llll|}
\hline Variable & group 1(n=68) & group 2(n=67) & P-value \\
\hline Age (years) (range) & $39.43 \pm 10.20(20-78)$ & $38.43 \pm 8.22(23-76)$ & 0.118 \\
\hline Gender (male/female) & $46 / 22$ & $41 / 26$ & 0.475 \\
\hline $\mathrm{BMI}\left(\mathrm{Kg} / \mathrm{m}^{2}\right)$ & $23.51 \pm 3.48$ & $22.99 \pm 3.16$ & 0.204 \\
\hline Stone size $\left(\mathrm{mm}^{2}\right)$ & $653.04 \pm 208.51$ & $649.34 \pm 233.15$ & 0.239 \\
\hline Stone position (left/right) & $29 / 39$ & $36 / 31$ & 0.230 \\
\hline
\end{tabular}

\subsection{Comparison of intraoperative and postoperative related indexes between the two groups}

The intraoperative and postoperative conditions of the two groups were shown in Table 2. There were no Loading [MathJax]/jax/output/CommonHTML/fonts/TeX/fontdata.js ;ition, hemoglobin decline, VAS score on the day 
of discharge, postoperative fever rate, and clear stone rate. No blood transfusion and interventional hemostasis were performed in either group. All patients had no pneumothorax or delayed bleeding. The stones under the guidance of B-ultrasound during surgery were completely removed. With respect to stone removal rates, differences between the two groups were not statistically significant $(P>0.05)$ after the first operation. The maximum diameter of all the residual stones was less than $4 \mathrm{~mm}$. The average postoperative hospital stay in group 2 was significantly longer than in group $1(P=0.004)$. There were significant differences in VAS scores between the two groups at $6 \mathrm{~h}$ postoperatively $(P=0.035)$ and on the first postoperative day $(P=0.034)$. However, there was no significant difference in VAS scores on the day of discharge $(P=0.211)$. And the two groups of patients were statistically significant in the use of analgesic drugs $(P=0.042)$.

Table 2

Comparison of intraoperative and postoperative related indexes between the two groups

\begin{tabular}{|llll|}
\hline Variable & group 1(n=68) & group 2(n=67) & $P$ value \\
\hline surgery time ( $\mathrm{min})$ & $69.91 \pm 14.59$ & $71.73 \pm 17.36$ & 0.055 \\
\hline postoperative hospital stay days( day) & $3.15 \pm 1.42$ & $6.48 \pm 1.88$ & 0.004 \\
\hline Piercing position & & & 0.391 \\
\hline 12 under the ribs & 38 & 32 & \\
\hline 11 intercostal space & 30 & 35 & 0.479 \\
\hline Hemoglobin decreased (g/L) & $1.87 \pm 0.94$ & $1.57 \pm 0.90$ & \\
\hline Postoperative VAS score & & & 0.035 \\
\hline 6 h after surgery & $4.07 \pm 1.79$ & $6.24 \pm 1.33$ & 0.034 \\
\hline day 1 postoperative & $2.50 \pm 1.76$ & $3.83 \pm 2.22$ & 0.211 \\
\hline on the day of discharge & $0.91 \pm 0.84$ & $1.15 \pm 0.93$ & 0.042 \\
\hline analgesics use $(\mathrm{n})$ & 6 & 15 & 0.713 \\
\hline fever(n) & 4 & 3 & 0.998 \\
\hline Stone removal rate $(\%)$ & $66 / 68(97.06)$ & $65 / 67(97.01)$ & \\
\hline
\end{tabular}

\section{Discussion}

With the concept of rapid rehabilitation surgery ${ }^{[7]}$, minimally invasive surgery has gradually been valued by surgeons. The choice of anesthesia is also an important part of rapid rehabilitation surgery. The kidney and ureter are subject to sympathetic and parasympathetic innervation. The sensory nerves follow the sympathetic and parasympathetic branches. The kidneys and ureters are not sensitive to cauterization and cutting. They are mainly sensitive to traction and increased ureteral pressure in the Loading [MathJax]/jax/output/CommonHTML/fonts/TeX/fontdata.js 
renal pelvis. The pain is generally dull, which may be associated with sympathetic stimulation, such as bloating, nausea, vomiting and so on. Because of these characteristics of the distribution of urinary nervous system, local anesthesia can meet the surgical requirements. Most hospitals use epidural anesthesia or general anesthesia for surgery. However, compared with epidural and general anesthesia, local anesthesia has the lowest complication and mortality ${ }^{[8]}$ in our hospital. Most patients underwent PCNL under local anesthesia, except for infants and young children. The results of this study show that PCNL under local anesthesia has the following advantages: Firstly, PCNL can avoid the bad impact on cardiopulmonary function of patients under local anesthesia. Secondly, pethidine hydrochloride has a good effect on internal organs pain, and lidocaine has a good effect on the pain caused by puncture ${ }^{[9]}$. Communicating with the patient during the operation can relieve the patient's nervousness and anxiety in time so that the patient can better tolerate and cooperate with the operation. In epidural anesthesia, due to visceral reflex pain, anesthesiologists are accustomed to use sedative drugs. Patients are often prone to restlessness, position changes which affect surgical operations. Thirdly, patients do not need enema and fasting before surgery and can eat immediately after surgery; Last but not least, PCNL under local anesthesia can reduce treatment costs and hospital stays. The results of this study show that PCNL has a wide range of indications under local anesthesia, and patients can tolerate except for infants and young children. The minimum age of this group is 20 years old and the maximum age is 78 years old. All patients have successfully completed the operation. Some patients who cannot use epidural anesthesia and general anesthesia can also perform PCNL in local anesthesia. In this group of,2 patients aged 78 years old were completed by PCNL under local anesthesiaDThe contraindications for PCNL under local anesthesia in addition to infants and young children are the same as conventional PCNL.

PCNL is the preferred method for the treatment of kidney stones and partial ureteral stones which were larger than $2 \mathrm{~cm}$ in diameter. In recent years, studies have reported the use of new technologies and improved protocols, such as minimally invasive PCNL, tubeless PCNL, and PCNL under local anesthesia to reduce postoperative analgesia and hospital stay ${ }^{[10,11]}$. The tubeless PCNL was first proposed by Bellman et al ${ }^{[12]}$ in 1997 . Since then, research reports on the safety and effectiveness of tubeless PCNL have emerged. There are some advantages of tubeless PCNL group over traditional PCNL including faster recovery, shorter hospital stays, lower hospitalization costs, less postoperative pain, less analgesic drugs usage ${ }^{[13]}$. But there was no difference between the two groups in postoperative complication. Recently, Shen and Zhong et al ${ }^{[14,15]}$ compared the tubeless PCNL with the common PCNL by meta-analysis, which confirmed that the postoperative analgesia requirement of the tubeless PCNL was lower and the hospital stay was shorter. And in recent years, the application of tubeless PCNL has become more and more extensive, including bilateral kidney stones and isolated kidney stones $[16,17]$.

The results of this study showed that the operation time of group 2 was slightly longer than the group 1 , which mainly because it took a certain time to place and fix the fistula. But the operation time between the two groups was not significantly different. In the postoperative hospital stay, group 2 was significantly longer than group 1 , which was consistent with the current study results. The postoperative 
sputum tube stimulates the intercostal or subcostal nerve; Second, The fistula tube produces irritation to the renal pelvis and renal pelvis wall. Pain after PCNL is generally mild, and most of them can be relieved without treatment or non-steroidal drugs. Only a small number of patients required narcotic analgesia. Group 2 patients with narcotic analgesic drugs were significantly more than group 1, the difference was statistically significant, and which was consistent with the VAS score. The reason why the postoperative hospital stay of group 1 was significantly shorter than that of group 2 was mainly because we routinely indwelled the fistula for $4 \sim 5$ days. Furthermore, after extubation, patients were generally observed after 1 day and then discharged. However, patients with untreated PCNL are usually discharged on the third day after surgery if they are asymptomatic. The removal of the renal fistula was generally performed without the fever, pain and urine leakage after the clamped tube, so the VAS score at the time of discharge was not significantly different between the two groups. There were no blood transfusion, interventional hemostasis, pneumothorax and delayed bleeding in all patients. With respect to puncture site, hemoglobin decline, postoperative fever rate between the two groups were not significantly different. No blood transfusion and interventional hemostasis were performed in either group, and no pneumothorax or delayed bleeding occurred in all patients.

In this study, 135 patients underwent mPCNL under local anesthesia with no serious complications and rapid postoperative recovery. This study showed that PCNL surgery under local anesthesia is simple, safe and effective, and worthy of clinical application. Tubeless MPCNL treatment of kidney stones can significantly reduce postoperative pain and discomfort and shorten hospital stays. But it is a retrospective study with possible selection bias, it is necessary to avoid some syndromes, such as intraoperative bleeding, ureteral obstruction, severe perforation of the collection system and residual stones.

\section{Conclusion}

Tubeless $\mathrm{mPCNL}$ is a simple, safe and effective technique under local anesthesia. It is worthy of clinical application. Tubeless mPCNL treatment for renal stones can significantly reduce postoperative pain and shorten hospital stays compared with common m PCNL.

\section{Abbreviations}

mPCNL:minimally invasive percutaneous nephrolithotomy; PCNL:percutaneous nephrolithotomy; VAS:visual analogue scale; IVU:intravenous urography; CSU:Central South University

\section{Declarations}

\section{Ethics approval and consent to participate}

This research project was reviewed and approved by our Member of Medical Ethics Audit Committee of

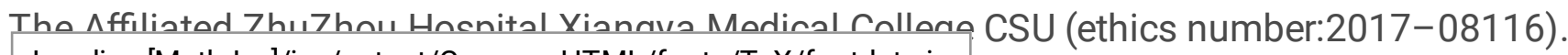




\section{Consent for publication}

Not applicable.

\section{Availability of data and materials}

The datasets used and analysed in the current study are available from the corresponding author upon reasonable request.

\section{Competing Interests}

The authors declare that they have no competing interests.

\section{Funding}

This work was funded by Natural Science Foundation of Hunan Province (\#2017JJ4067). Funders were not involved in the design of the study, collecting and analyzing data, and writing and reviewing papers.

\section{Authors' contributions}

G.Y. Qu, wrote the main manuscript text. All the authors reviewed the manuscript and discussed the results and edited the manuscript.

\section{Conflict of Interest}

1. Research involving Human Participants and/or Animals: All procedures performed in studies involving human participants were in accordance with the ethical standards of the institutional and/or national research committee and with the 1964 Helsinki declaration and its later amendments or comparable ethical standards.

2. Informed consent: Informed consent was obtained from all individual participants included in the study.

\section{Acknowledgement}

Thanks to all urologists, anesthesiologists and nurses who participated in this study in The Affiliated ZhuZhou Hospital Xiangya Medical College CSU. 


\section{References}

1. Ziemba JB, Matlaga BR. Guideline of guidelines: kidney stones [J]. BJU Int. 2015;116(2):184-9. doi:10.1111/bju.13080.

2. Moosanejad N, Firouzian A, Hashemi SA, et al. Comparison of totally tubeless percutaneous nephrolithotomy and standard percutaneous nephrolithotomy for kidney stones: a randomized, clinical trial [J]. Braz J Med Biol Res. 2016;49(4):e4878. doi:10.1590/1414-431x20154878.

3. Kirac M, Tepeler A, Bozkurt OF, et al. The efficacy of bupivacaine infiltration on the nephrostomy tract in tubeless and standard percutaneous nephrolithotomy: a prospective, randomized, multicenter study [J]. Urology. 2013;82(3):526-31. doi:10.1016/j.urology.2013.02.083.

4. Gonulalan $\mathrm{U}$, Cicek $\mathrm{T}$, Istanbulluoglu $\mathrm{O}$, et al. Tubeless percutaneous nephrolithotomy is effective and safe in short- and long-term urinary drainage [J]. Urolithiasis. 2013;41(4):341-6. doi:10.1007/s00240-013-0560-0.

5. Kuzgunbay B, Turunc T, Akin S, et al. Percutaneous nephrolithotomy under general versus combined spinal-epidural anesthesia [J]. J Endourol. 2009;23(11):1835-8. doi:10.1089/end.2009.0261.

6. Singh V, Sinha RJ, Sankhwar SN, et al. A prospective randomized study comparing percutaneous nephrolithotomy under combined spinal-epidural anesthesia with percutaneous nephrolithotomy under general anesthesia [J]. Urol Int. 2011;87(3):293-8. doi:10.1159/000329796.

7. Kehlet $\mathrm{H}, \mathrm{Dahl} \mathrm{JB}$. Anaesthesia, surgery, and challenges in postoperative recovery [J]. Lancet. 2003;362(9399):1921-8. doi:10.1016/s0140-6736(03)14966-5.

8. Ecke TH, Barski D, Weingart G, et al. Presentation of a method at the Exploration Stage according to IDEAL: Percutaneous nephrolithotomy (PCNL) under local infiltrative anesthesia is a feasible and effective method - retrospective analysis of 439 patients [J]. Int J Med Sci. 2017;14(4):302-9. doi:10.7150/ijms.17963.

9. Giannakopoulos S, Giannopoulos S, Gardikis S, et al. Second-look Flexible Nephroscopy Combined With Holmium: Yttrium-Aluminum-Garnet Laser Lithotripsy Under Local Anesthesia: A Prospective Study [J]. Urology, 2017, 9927-32. doi:10.1016/j.urology.2016.06.074.

10. Tangpaitoon T, Nisoog C, Lojanapiwat B. Efficacy and safety of percutaneous nephrolithotomy (PCNL): a prospective and randomized study comparing regional epidural anesthesia with general anesthesia [J]. Int Braz J Urol. 2012;38(4):504-11.

11. Sourial MW, Francois N, Box GN, et al. Supracostal access tubeless percutaneous nephrolithotomy: minimizing complications [J]. World J Urol. 2018. doi:10.1007/s00345-018-2518-x.

12. Bellman GC, Davidoff R, Candela J, et al. Tubeless percutaneous renal surgery [J]. J Urol. 1997;157(5):1578-82.

13. Tirtayasa PMW, Yuri P, Birowo P, et al. Safety of tubeless or totally tubeless drainage and nephrostomy tube as a drainage following percutaneous nephrolithotomy: A comprehensive review [J]. Asian J Surg. 2017;40(6):419-23. doi:10.1016/j.asjsur.2016.03.003. 
14. Shen P, Liu Y, Wang J. Nephrostomy tube-free versus nephrostomy tube for renal drainage after percutaneous nephrolithotomy: a systematic review and meta-analysis [J]. Urol Int. 2012;88(3):298306. doi:10.1159/000332151.

15. Zhong Q, Zheng C, Mo J, et al. Total tubeless versus standard percutaneous nephrolithotomy: a meta-analysis [J]. J Endourol. 2013;27(4):420-6. doi:10.1089/end.2012.0421.

16. Choi SW, Kim KS, Kim JH, et al. Totally tubeless versus standard percutaneous nephrolithotomy for renal stones: analysis of clinical outcomes and cost [J]. J Endourol. 2014;28(12):1487-94. doi:10.1089/end.2014.0421.

17. Kokorovic A, Wilson JW, Beiko D. Outpatient bilateral supracostal tubeless percutaneous nephrolithotomy for staghorn calculi [J]. Can Urol Assoc J. 2014;8(3-4):E273-5. doi:10.5489/cuaj.1691.

\section{Unsectioned Tables}

\begin{tabular}{|llll|}
\hline Variable & group 1(n=68) & group 2(n=67) & P-value \\
\hline Age (years) (range) & $39.43 \pm 10.20(20-78)$ & $38.43 \pm 8.22(23-76)$ & 0.118 \\
\hline Gender (male/female) & $46 / 22$ & $41 / 26$ & 0.475 \\
\hline $\mathrm{BMI}\left(\mathrm{Kg} / \mathrm{m}^{2}\right)$ & $23.51 \pm 3.48$ & $22.99 \pm 3.16$ & 0.204 \\
\hline Stone size $\left(\mathrm{mm}^{2}\right)$ & $653.04 \pm 208.51$ & $649.34 \pm 233.15$ & 0.239 \\
\hline Stone position (left/right) & $29 / 39$ & $36 / 31$ & 0.230 \\
\hline
\end{tabular}




\begin{tabular}{|llll|}
\hline Variable & group 1(n=68) & group 2(n=67) & P value \\
\hline surgery time / min & $69.91 \pm 14.59$ & $71.73 \pm 17.36$ & 0.055 \\
\hline postoperative hospital stay days / d & $3.15 \pm 1.42$ & $6.48 \pm 1.88$ & 0.004 \\
\hline Piercing position & & & 0.391 \\
\hline 12 under the ribs & 38 & 32 & \\
\hline 11 intercostal space & 30 & 35 & 0.479 \\
\hline Hemoglobin decreased (g/L) & $1.87 \pm 0.94$ & $1.57 \pm 0.90$ & \\
\hline Postoperative VAS score & & & 0.035 \\
\hline 6 h after surgery & $4.07 \pm 1.79$ & $6.24 \pm 1.33$ & 0.034 \\
\hline day 1 postoperative & $2.50 \pm 1.76$ & $3.83 \pm 2.22$ & 0.211 \\
\hline on the day of discharge & $0.91 \pm 0.84$ & $1.15 \pm 0.93$ & 0.042 \\
\hline analgesics use $(\mathrm{n})$ & 6 & 15 & 0.713 \\
\hline fever(n) & 4 & 3 & 0.998 \\
\hline Stone removal rate $(\%)$ & $66 / 68(97.06)$ & $65 / 67(97.01)$ & \\
\hline
\end{tabular}

\section{Supplementary Files}

This is a list of supplementary files associated with this preprint. Click to download.

- ApplicationFormofEthicalapproval.doc 